\title{
The Potential Long-Term Comparative Effectiveness of Larotrectinib and Entrectinib for Second-Line Treatment of TRK Fusion-Positive Metastatic Lung Cancer
}

\author{
Joshua A. Roth, PhD, MHA; Josh J. Carlson, PhD; Fang Xia, PhD; \\ Todd Williamson, PhD; and Sean D. Sullivan, PhD
}

\begin{abstract}
BACKGROUND: Larotrectinib and entrectinib are FDA-approved therapies for patients with non-small cell lung cancer (NSCLC) with neurotrophic receptor tyrosine kinase gene fusion (TRK fusion-positive) whose cancer has metastasized and progressed. Early evidence indicates that these targeted therapies may offer dramatic survival benefits versus traditional cytotoxic regimens, but it remains uncertain how larotrectinib and entrectinib compare with each other.
\end{abstract}

OBJECTIVE: To simulate and compare expected life-years and qualityadjusted life-years (QALYs) for both TRK inhibitors.

METHODS: We developed a partitioned survival model to project the longterm comparative effectiveness of larotrectinib versus entrectinib in second-line treatment of metastatic NSCLC. Larotrectinib survival data were derived from a 13-month follow-up of 12 patients with TRK fusion-positive NSCLC in the NCT02122913 (phase 1) and NCT02576431 (NAVIGATE) trials. Entrectinib survival data were derived from a 13-month follow-up of 10 patients with TRK fusion-positive NSCLC in the ALKA-372-001, STARTRK-1, and STARTRK-2 trials. For larotrectinib and entrectinib progression-free survival and overall survival (OS), in-trial survival was extrapolated using parametric curve fits. Exponential fits were selected for all survival models based on minimal Bayesian information criteria and clinical plausibility. Lifetime survival curves were used to estimate expected mean/median survival. QALYs were estimated by applying preprogression and postprogression health state utilities derived from the literature.

RESULTS: In the base case, treatment with larotrectinib and entrectinib resulted in 5.4 and 1.2 median preprogression life-years and 7.0 and 1.8 median total life-years, respectively. Mean preprogression life-years (QALYs) were 7.5 (5.0) and 1.9 (1.2), and mean total life-years (QALYs) were $9.2(5.8)$ and $4.4(2.4)$, respectively.

CONCLUSIONS: Among TRK inhibitors for metastatic NSCLC, larotrectinib is estimated to provide improved life-year and QALY outcomes versus entrectinib based on parametric extrapolations of in-trial survival data. Our analysis is limited by lack of NSCLC-specific data on entrectinib OS, the small samples of patients with NSCLC in the trials, and a cross-trial comparison. Future studies should re-evaluate the comparative effectiveness of larotrectinib versus entrectinib as more patients are treated and as longterm survival data mature.

J Manag Care Spec Pharm. 2020;26(8):981-86

Copyright $\odot 2020$, Academy of Managed Care Pharmacy. All rights reserved.

\section{What is already known about this subject}

Early evidence indicates that the receptor tyrosine kinase (TRK) inhibitors larotrectinib and entrectinib may offer dramatic survival benefits versus traditional cytotoxic regimens for treatment in TRK fusion-positive metastatic non-small cell lung cancer (NSCLC).

No study has evaluated the potential comparative effectiveness of larotrectinib versus entrectinib for second-line treatment of TRK fusion-positive metastatic NSCLC.

\section{What this study adds}

We used larotrectinib and entrectinib clinical trial data and simulation modeling to project the potential long-term comparative effectiveness of the 2 FDA-approved TRK inhibitors for treatment in TRK fusion-positive metastatic NSCLC

We found that larotrectinib is expected to provide improved lifeyear and quality-adjusted life-year outcomes compared with entrectinib based on parametric extrapolations of in-trial survival data.

Future studies should reevaluate the comparative effectiveness of larotrectinib and entrectinib as greater numbers of patients are treated and as long-term survival data mature.

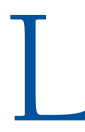

arotrectinib and entrectinib are therapies approved by the U.S. Food and Drug Administration (FDA) for adult patients with neurotrophic receptor tyrosine kinase gene fusion (TRK fusion-positive) found in multiple solid tumor types whose cancer has metastasized and progressed. Entrectinib also was approved for treatment in adults with non-small cell lung cancer (NSCLC) with ROS1 fusion-positive tumors. Emerging evidence indicates that these targeted therapies may offer potentially dramatic survival benefits for patients compared with traditional cytotoxic regimens. ${ }^{1,2}$

Guidelines recommend that patients who initiate on either larotrectinib or entrectinib do not use the alternate TRK inhibitor as a subsequent line of treatment, that is, clinicians and patients need to decide between these 2 products for their only chance at using a TRK inhibitor. ${ }^{3}$ However, data on larotrectinib and entrectinib comparative effectiveness and safety remain uncertain, in part because of the design of the clinical 
research programs used to support FDA approval. Both clinical research programs employed single-arm, basket trial designs without concurrent or historical control groups in relatively small populations of patients. ${ }^{1,2}$ The trial populations were relatively similar across key characteristics, and the available data to date indicate longer median progression-free (PFS) and overall survival (OS) in samples of all patients treated across tumor types with larotrectinib (median PFS $=28.3$ months, median OS $=44.4$ months) as compared with entrectinib (median $\mathrm{PFS}=11.2$ months, median OS=20.9 months). ${ }^{1,2}$ In small samples of patients with NSCLC, larotrectinib median PFS and OS were not reached $(n=12)$, while entrectinib median PFS was 14.9 months, and OS results have not been reported $(n=10)$.

Of importance to clinicians and decision makers is that extrapolation of within-trial survival outcomes using standard parametric curve fit methods can provide more comprehensive estimates of the potential comparative effectiveness of larotrectinib and entrectinib in treatment for NSCLC and can allow for estimation on mean life expectancy and quality-adjusted lifeyears (QALYs). ${ }^{4}$ The objective of this study was to use available evidence to extrapolate and compare expected life-years and QALYs for both TRK inhibitors in metastatic NSCLC to inform initial stakeholder considerations about potential comparative effectiveness.

\section{Methods}

\section{Approach Overview}

We developed a partitioned survival simulation model in Microsoft Excel (version 16.16.12, Redmond, WA) to project long-term comparative effectiveness of larotrectinib and entrectinib in second-line treatment of metastatic NSCLC. ${ }^{5}$ The simulation evaluated preprogression and OS over a lifetime horizon (maximum 50-year follow-up, to age 102 years). The model was populated with larotrectinib and entrectinib survival, response, and health-related quality of life data drawn from several clinical trials reported at recent major oncology conferences. It should be noted that all source studies had limited sample size $(\mathrm{n}<60)$ and median follow-up duration (13 months). ${ }^{2,6}$ We modeled parametric curve fits to in-trial PFS and OS data to project long-term survival outcomes., ${ }^{4,7}$ Outcomes included expected $3-, 5-$, and 10-year survival proportions as well as mean expected life-years over a lifetime horizon.

\section{Larotrectinib Survival Data Sources}

Larotrectinib OS and PFS data were derived from a 13-month follow-up of 12 patients with TRK fusion-positive NSCLC in the NCT02122913 (phase 1) and NCT02576431 (NAVIGATE) trials. $^{2}$ Among patients in this sample, median age was 52.0 years; $50 \%(n=6)$ were female; $92 \%(n=11)$ had $\geq 1$ previous systemic therapy; $50 \%(n=6)$ had 3 or more systemic therapies; and $8 \%(n=1)$ had no previous systemic therapy.

\section{Entrectinib Survival Data Sources}

Entrectinib NSCLC PFS data were derived from a 13-month follow-up of 10 patients with TRK fusion-positive NSCLC in the ALKA-372-001, STARTRK-1, and STARTRK-2 trials. ${ }^{\circ}$ Among patients in this sample, median age was 62.5 years, $50 \%(n=5)$ were female, $30 \%(n=3)$ had 1 previous systemic therapy, $40 \%(n=4)$ had 2 or more systemic therapies, and 30\% ( $n=3)$ had no previous systemic therapy.

While there have been reports of entrectinib OS pooled across TRK fusion-positive tumor types, there have not been reports of entrectinib OS data in the subgroup of patients with TRK fusion-positive NSCLC. To model survival in the absence of such data, we imputed entrectinib OS by applying the same monthly ratio of OS to PFS observed in patients receiving larotrectinib to entrectinib PFS outcomes in ALKA-372-001, STARTRK-1, and STARTRK-2 trials. ${ }^{6}$ This approach resulted in a mean of 4.38 expected life years-74\% greater than the mean life-years estimated from the model (2.52) when OS was derived from pooled entrectinib results across 54 patients with any TRK fusion-positive tumor type in the ALKA-372-001, STARTRK-1, and STARTRK-2 trials and extrapolated with an exponential curve fit.

\section{Parametric Survival Curve Fits}

In-trial survival outcomes were extrapolated using parametric curve fits (Weibull, exponential, log-logistic, gamma) for larotrectinib PFS and OS as well as entrectinib OS. ${ }^{7}$ For entrectinib PFS, an exponential model was fit to the median PFS estimate for 10 patients with TRK fusion-positive NSCLC. This approach was taken in the absence of publicly reported Kaplan-Meier survival curves showing monthly survival proportions over the 13-month follow-up period.

Based on minimal Bayesian information criteria, clinical plausibility, and visual inspection, exponential fits were selected to simulate long-term survival in all PFS and OS survival models. Lifetime survival curves were used to estimate expected mean and median PFS and OS. Lifetime survival curves were designed so that survival age-based mortality rates could not be lower than those for the general public as reported in U.S. life tables. ${ }^{8}$ If parametric curve fit annual mortality rates were less than that of U.S. life tables for a given mean age of the cohort, then mortality rates reverted to those of the life tables.

\section{Health State Utility Values for Estimation of QALYs}

Health state utility values for preprogression and postprogression health states ( 0.66 and 0.47 , respectively) were derived from a societal based valuation study of 100 people in the United Kingdom using standard gamble methods. ${ }^{9}$ These values were applied to survival time in preprogression and postprogression states to estimate expected QALYs over a lifetime horizon. 
The Potential Long-Term Comparative Effectiveness of Larotrectinib and

Entrectinib for Second-Line Treatment of TRK Fusion-Positive Metastatic Lung Cancer

\section{FIGURE 1 Parametric Extrapolations of Larotrectinib and Entrectinib Overall and Progression-Free Survival}

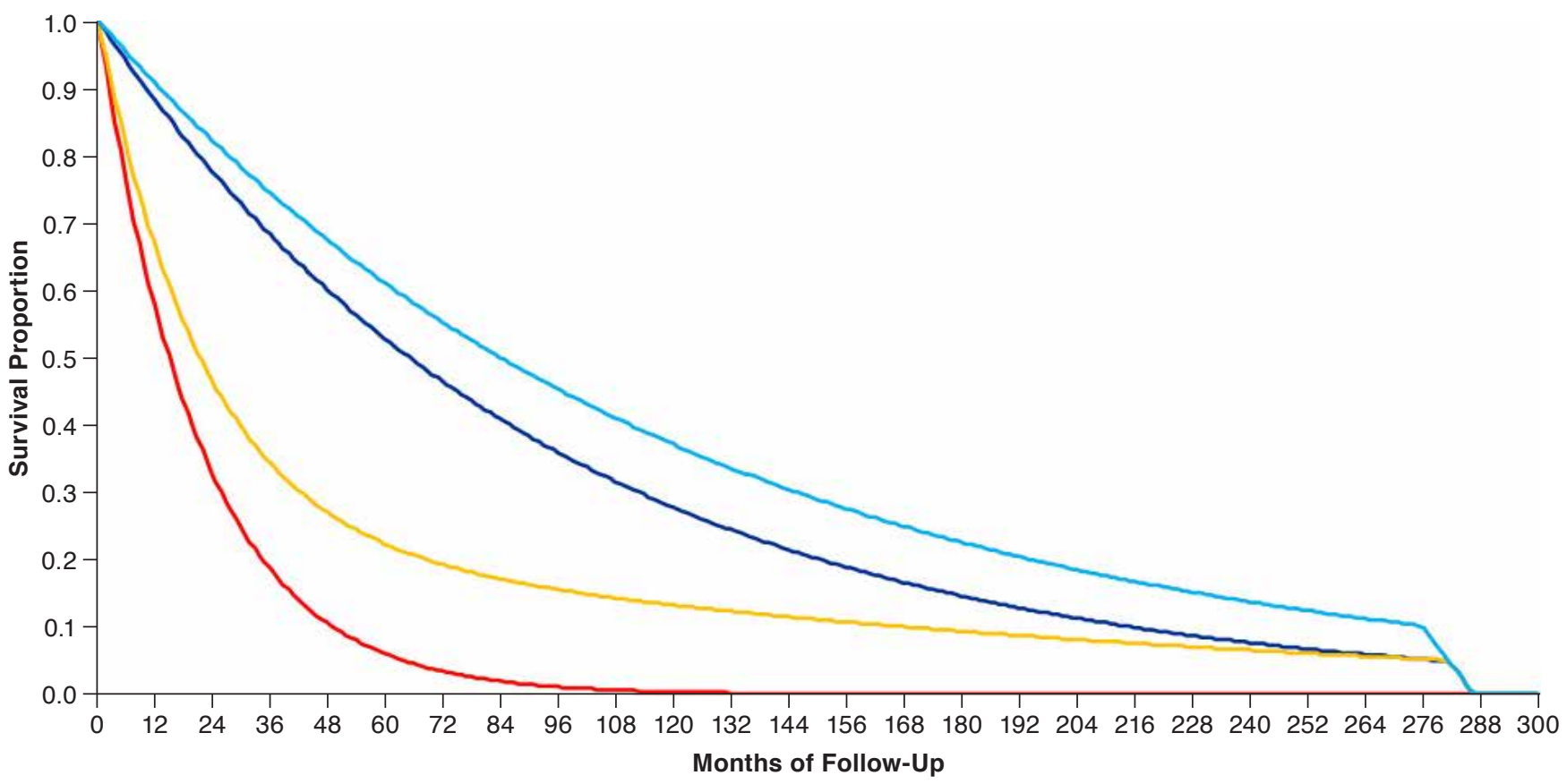

$$
\begin{array}{ll}
- \text { Entrectinib progression-free survival } & \text { Larotrectinib progression-free survival } \\
\text { Entrectinib overall survival } & \text { Larotrectinib overall survival }
\end{array}
$$

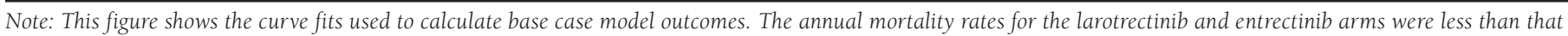

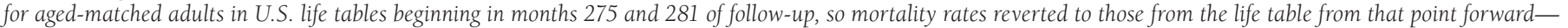
consequently, the sharply declining curves.

\section{Scenario Analyses}

Due to the small samples of patients with NSCLC in the larotrectinib $(n=12)$ and entrectinib $(n=10)$ trials and the limited median follow-up duration of 13 months, there was a high degree of uncertainty about long-term survival with both therapies. As such, we undertook sensitivity analyses with wide uncertainty ranges to explore the implications of alternative survival outcomes that might be demonstrated with larger samples tracked over longer follow-up periods. Specifically, we evaluated survival and QALY outcomes in scenarios where (a) larotrectinib OS hazard was increased by 30\% (i.e., less favorable survival) versus the base case larotrectinib OS function, and (b) entrectinib OS was decreased by 30\% (i.e., more favorable survival) versus the base case entrectinib OS function. The magnitude of variation in survival functions $( \pm 30 \%)$ was arbitrarily selected as a large but statistically plausible deviation in survival outcomes as compared with the base case. We took this approach in absence of reported 95\% confidence intervals for survival outcomes. Furthermore, it would be expected that survival function confidence intervals derived from samples of 10 or 12 patients that could have highly variable survival durations would result in such extreme values that they would not be informative in our sensitivity analyses.

Using the sensitivity analyses survival functions previously described, we conducted 3 additional pairwise comparisons of larotrectinib and entrectinib: (1) sensitivity analysis of larotrectinib survival versus base case entrectinib survival, (2) base case larotrectinib survival versus sensitivity analysis of entrectinib survival, and (3) sensitivity analysis of larotrectinib survival versus sensitivity analysis of entrectinib survival. These comparisons would assess (a) comparative effectiveness under less favorable larotrectinib survival, (b) comparative effectiveness under more favorable entrectinib survival, and (c) comparative effectiveness under an extreme scenario with less favorable larotrectinib survival and more favorable entrectinib survival, respectively. We conducted these analyses recognizing the possibility that if all resulted in positive incremental survival outcomes for larotrectinib, it could reinforce the likely favorable comparative effectiveness of larotrectinib 
The Potential Long-Term Comparative Effectiveness of Larotrectinib and Entrectinib for Second-Line Treatment of TRK Fusion-Positive Metastatic Lung Cancer

\begin{tabular}{|c|c|c|c|c|c|c|}
\hline \multirow[b]{2}{*}{ Scenario } & \multicolumn{2}{|c|}{ Larotrectinib Outcomes } & \multicolumn{2}{|c|}{ Entrectinib Outcomes } & \multicolumn{2}{|c|}{ Difference in Outcomes } \\
\hline & Preprogression & Total & Preprogression & Total & Preprogression & Total \\
\hline Base case & $7.49 \quad(5.00)$ & $9.15 \quad(5.78)$ & $1.85 \quad(1.23)$ & $4.38 \quad(2.43)$ & $5.64 \quad(3.77)$ & (3.36) \\
\hline $\begin{array}{l}\text { Alternative scenario 1: larotrectinib 30\% } \\
\text { increase in PFS and OS hazard }\end{array}$ & $6.02 \quad(4.02)$ & $7.44 \quad(4.69)$ & $1.85 \quad(1.23)$ & $4.38 \quad(2.43)$ & $4.17 \quad(2.79)$ & $3.05 \quad(2.27)$ \\
\hline $\begin{array}{l}\text { Alternative scenario 2: entrectinib 30\% } \\
\text { decrease in PFS and OS hazard }\end{array}$ & $7.49 \quad(5.00)$ & $9.15 \quad(5.78)$ & $2.56 \quad(1.70)$ & $6.77 \quad(3.75)$ & $4.93 \quad(3.30)$ & $2.38 \quad(2.03)$ \\
\hline $\begin{array}{l}\text { Alternative scenario 3: combination of } \\
\text { scenarios } 1 \text { and } 2\end{array}$ & $6.02 \quad(4.02)$ & $7.44 \quad(4.69)$ & $2.56 \quad(1.70)$ & $6.77 \quad(3.75)$ & $3.46 \quad(2.32)$ & $0.67 \quad(0.95)$ \\
\hline
\end{tabular}

versus entrectinib in the treatment for TRK fusion-positive NSCLC that would likely be demonstrated in the base case.

\section{Results}

\section{Base Case Survival Results}

In the base case, treatment with larotrectinib and entrectinib resulted in a median of 5.4 and 1.3 preprogression life-years and a median of 7.0 and 1.8 total-life years, respectively (Figure 1). Mean preprogression life-years were 7.5 and 1.9, and mean total life-years were 9.2 and 4.4 , respectively (Table 1). Projected OS proportions at 3,5, and 10 years were $75 \%, 61 \%$, and $37 \%$ with larotrectinib, and 35\%, 22.4\%, and $13.2 \%$ with entrectinib (Figure 1).

\section{Base Case Quality-Adjusted Survival Results}

In the base case, treatment with larotrectinib and entrectinib resulted in mean preprogression QALYs of 5.0 and 1.2 and mean total QALYs of 5.8 and 2.4, respectively (Table 1).

\section{Sensitivity Analysis Survival Results}

Scenario 1: Sensitivity Analysis of Larotrectinib Survival (-30\%) Versus Base Case Entrectinib Survival. In scenario 1, treatment with larotrectinib and entrectinib resulted in 5-year survival proportions of $53 \%$ and $22 \%$, respectively. Mean preprogression life-years were 6.0 and 1.9, and mean QALYs were 4.0 and 1.2 , respectively (Table 1 ). Mean total life-years were 7.4 and 4.4, and mean QALYs were 4.7 and 2.4, respectively (Table 1).

Scenario 2: Base Case Larotrectinib Survival Versus Sensitivity Analysis of Entrectinib Survival (+30\%). In scenario 2, treatment with larotrectinib and entrectinib resulted in 5-year survival proportions of $61 \%$ and $35 \%$, respectively. Mean preprogression life-years were 7.5 and 2.6, and mean QALYs were 5.0 and 1.7, respectively (Table 1). Mean total life-years were 9.2 and 6.8, and mean QALYs were 5.8 and 3.7, respectively (Table 1 ).
Scenario 3: Sensitivity Analysis of Larotrectinib Survival (-30\%) Versus Sensitivity Analysis of Entrectinib Survival $(+30 \%)$. In scenario 3, treatment with larotrectinib and entrectinib resulted in 5-year survival proportions of 53\% and $35 \%$, respectively. Mean preprogression life-years were 6.0 and 2.6, and mean QALYs were 4.0 and 1.7, respectively (Table 1). Mean total life-years were 7.4 and 6.8, and mean QALYs were 4.7 and 3.7 , respectively (Table 1 ).

\section{Discussion}

Using emerging clinical data, we set out to develop comparative effectiveness estimates of larotrectinib and entrectinib in patients with TRK fusion-positive metastatic NSCLC. We undertook this analysis to better inform clinical and health care system reimbursement decisions, in part because both of these targeted, tumor-agnostic agents were FDA-approved with very limited clinical evidence.

We found that among TRK fusion protein-targeted therapies for metastatic NSCLC, larotrectinib is estimated to provide improved life-year and QALY outcomes versus entrectinib using extrapolations of in-trial survival data. Larotrectinib survival gains remain highly uncertain, but scenario analyses suggested that gains are expected to persist under a range of clinically plausible survival effects. Future studies should re-evaluate the comparative outcomes of larotrectinib and entrectinib as more patients are treated, survival data mature, and real-world evidence studies are conducted in TRK fusionpositive NSCLC.

In the past 20 years, the treatment landscape in advanced NSCLC has evolved from a few traditional chemotherapeutic options with limited effectiveness to now include many innovative products ranging from immunotherapeutics to agents targeted to a variety of tumors molecular profiles. ${ }^{3}$ Five years ago, patients with TRK fusion-positive tumors were limited to untargeted products. With the arrival of the first TRK inhibitors, larotrectinib and entrectinib, these patients can potentially achieve unprecedented response rates and survival outcomes. ${ }^{2,6}$ However, the choice between TRK inhibitors is 
critical as guidelines recommend that patients receive only 1 of the 2 available treatment options. Given the evidence available in the peer-reviewed literature and conference proceedings, coupled with the estimates provided here, larotrectinib may be the optimal treatment option for patients who are TRK fusionpositive who have no satisfactory alternative treatments or that have progressed following cytotoxic treatment.

\section{Limitations}

Our analysis has several important limitations that should be noted. First, in-trial survival data were derived from small samples tracked for a median of 13 months. ${ }^{2,6}$ As such, there is a high degree of uncertainty about how well survival data from these samples will generalize to larger samples of patients with TRK fusion-positive NSCLC treated with larotrectinib or entrectinib. Recognizing this issue, we conducted a series of sensitivity analyses that widely varied the OS hazard functions for each agent and found that larotrectinib was still expected to result in a potentially clinically meaningful gain in survival versus entrectinib even if the larotrectinib hazard was increased by $30 \%$ and the entrectinib hazard was decreased by $30 \%$.

Second, there is limited publicly reported data about OS outcomes in patients with TRK fusion-positive NSCLC treated with entrectinib. To deal with this, we imputed entrectinib OS by applying the same monthly ratio of OS to PFS that was modeled for larotrectinib to the parametric extrapolation of in-trial PFS for entrectinib. This approach closely replicated reported OS in a pooled sample of TRK fusion-positive tumors treated with entrectinib and is also expected to minimize differences in comparative survival between larotrectinib versus entrectinib-an important factor to mitigate bias in our incremental analyses. ${ }^{6}$

Finally, this study involves a naive, direct (cross-trial) comparison of survival with larotrectinib and entrectinib. As samples were not derived from a single randomized trial, there is possible imbalance in prognostic factors between samples. For example, median age in the larotrectinib sample (52.0 years) was lower than median age in the entrectinib sample (62.5 years). This imbalance may account for some fraction of the more favorable survival we demonstrated for larotrectinib versus entrectinib. Still, given the relatively short survival duration of all patients diagnosed with advanced NSCLC, this imbalance likely only explains a limited amount of the mean survival differences we estimated. For example, U.S. life tables for the general public demonstrate that mortality rates for persons aged 52 years is only 1.09-fold greater than those aged 62 years, and our sensitivity analyses examined incremental outcomes under much larger uncertainty ranges. ${ }^{8}$ It is also possible that there are unmeasured prognostic factors that could vary between samples, which could impact comparative effectiveness estimates.
Our results should be interpreted with these notes in mind and comparative effectiveness should be reassessed using rigorous observational research designs as each agent has increased real-world use in clinical practice.

\section{Conclusions}

Among TRK inhibitors for treatment of metastatic NSCLC, larotrectinib is estimated to provide improved life-year and QALY outcomes compared with entrectinib based on parametric extrapolations of in-trial survival data. Our analysis is limited by a paucity of NSCLC-specific data on entrectinib OS; the small sample size of patients with NSCLC in source trials; and a naive, direct (cross-trial) comparison. Future studies should re-evaluate the comparative effectiveness of larotrectinib and entrectinib as greater numbers of patients are treated and as long-term survival data mature.

\section{Authors}

JOSHUA A. ROTH, PhD, MHA, Hutchinson Institute for Cancer Outcomes Research and Public Health Sciences Division, Fred Hutchinson Cancer Research Center, Seattle, Washington. JOSH J. CARLSON, PhD, CHOICE Institute, School of Pharmacy, University of Washington, Seattle. FANG XIA, PhD, and TODD WILLIAMSON, PhD, Bayer Healthcare, Parsippany, New Jersey. SEAN D. SULLIVAN, PhD, Hutchinson Institute for Cancer Outcomes Research, Fred Hutchinson Cancer Research Center, Seattle, Washington, and CHOICE Institute, School of Pharmacy, University of Washington, Seattle.

AUTHOR CORRESPONDENCE: Joshua A. Roth, PhD, MHA, Associate Member, Hutchinson Institute for Cancer Outcomes Research, Fred Hutchinson Cancer Research Center, 1101 Fairview Ave., N., Seattle, WA 98109. Tel.: 206.667.7867; Email:jroth@fredhutch.org.

\section{DISCLOSURES}

Funding for this study was contributed by Bayer Healthcare, which reviewed the manuscript drafts, and employees contributed to the manuscript as coauthors. Xia and Williamson are employees of Bayer Healthcare. Roth, Carlson, and Sullivan are consultants to Bayer Healthcare and retain rights to all final revisions to the manuscript. Carlson also reports fees from Adaptive Biotechnologies, unrelated to this work. Roth reports consulting fees from BMS, unrelated to this work.

\section{REFERENCES}

1. Hong DS, DuBois SG, Kummar S, et al. Larotrectinib in patients with TRK fusion-positive solid tumours: a pooled analysis of three phase $1 / 2$ clinical trials. Lancet Oncol. 2020;21(4):531-40.

2. Hyman DM, van Tilburg CM, Albert CM, et al. 445PD Durability of response with larotrectinib in adult and pediatric patients with TRK fusion cancer [abstract]. Ann Oncol. 2019;30(Suppl 5):vl62-63. Available at: https://www.annalsofoncology.org/article/S0923-7534(19)58667-7/fulltext. Accessed April 21, 2020. 
The Potential Long-Term Comparative Effectiveness of Larotrectinib and Entrectinib for Second-Line Treatment of TRK Fusion-Positive Metastatic Lung Cancer

3. Ettinger DS, Wood DE, Aggarwal C, et al. NCCN Guidelines Insights: Non-Small Cell Lung Cancer, Version 1.2020. J Natl Compr Canc Netw. 2019;17(12):1464-72. Available at: https://jnccn.org/view/journals/ jnccn/17/12/article-p1464.xml. Accessed April 15, 2020.

4. Jackson C, Stevens J, Ren S, et al. Extrapolating survival from randomized trials using external data: a review of methods. Med Decis Making. 2017;37(4):377-90

5. Glasziou PP, Simes RJ, Gelber RD. Quality adjusted survival analysis. Stat Med. 1990;9(11):1259-76.

6. Paz-Ares L, Doebele RC, Farago AF, et al. 113OEntrectinib in NTRK fusion-positive non-small cell lung cancer (NSCLC): integrated analysis of patients (pts) enrolled in STARTRK-2, STARTRK-1 and ALKA-372-001. Ann Oncol. 2019;30(Suppl 2):ii48-49.
7. Hoyle MW, Henley W. Improved curve fits to summary survival data: application to economic evaluation of health technologies. BMC Med Res Methodol. 2011;11:139.

8. Centers for Disease Control and Prevention. United States life tables, 2016. Natl Vital Stat Rep. 2019;68(4):1-65.

9. Nafees B, Stafford M, Gavriel S, Bhalla S, Watkins J. Health state utilities for non small cell lung cancer. Health Qual Life Outcomes. 2008;6:84. 